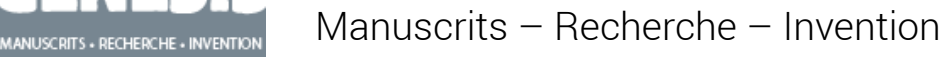

$50 \mid 2020$

Aragon

\title{
Du manuscrit de poème aux germes du roman : graines, pistes et restes
}

\section{Maryse Vassevière}

\section{(2) OpenEdition}

1 Journals

Édition électronique

URL : https://journals.openedition.org/genesis/5233

DOI : 10.4000/genesis.5233

ISSN : 2268-1590

Éditeur :

Presses universitaires de Paris Sorbonne (PUPS), Société internationale de génétique artistique littéraire et scientifique (SIGALES)

Édition imprimée

Date de publication : 15 juillet 2020

Pagination : 69-82

ISBN : 979-10-231-0679-4

ISSN : 1167-5101

\section{Référence électronique}

Maryse Vassevière, «Du manuscrit de poème aux germes du roman : graines, pistes et restes »,

Genesis [En ligne], 50 | 2020, mis en ligne le 15 juillet 2021, consulté le 03 septembre 2021. URL:

http://journals.openedition.org/genesis/5233 ; DOI : https://doi.org/10.4000/genesis.5233

Tous droits réservés 


\title{
Du manuscrit de poème aux germes du roman : graines, pistes et restes
}

\author{
Maryse Vassevière
}

$\mathrm{E}$ n léguant ses manuscrits au CNRS en 1977, Aragon invitait les chercheurs à l'exploration génétique de ses textes. Ces milliers de documents ont été classés en dossiers par les soins des chercheurs du fonds Triolet-Aragon du CNRS ${ }^{1}$ et ils sont maintenant conservés au département des Manuscrits de la BnF. Même si tous ces dossiers constitués pour chaque œuvre ne sont pas de même importance - absents pour certaines comme Les Cloches de Bâle, très fragmentaires pour les recueils de la poésie de la Résistance, ou au contraire très complets pour les romans (Aurélien, Les Communistes, Théâtrel Roman) et les grands recueils poétiques comme Le Roman inachevé ou Le Fou d'Elsa -, il est toujours passionnant de s'aventurer dans ce continent des manuscrits d'Aragon. J'ai pour ma part, à plusieurs reprises, tenté cette aventure sur les dossiers génétiques des romans et il me reviendra ici de poursuivre cette exploration en ouvrant ma recherche sur l'écriture poétique tout en me situant dans la lignée de mes précédentes recherches sur Aurélien, Théâtre/Roman et Les Incipit ${ }^{2}$... Centré sur un des rares manuscrits de travail de la poésie de la Résistance, cet article pourrait être une nuance apportée à l'image d'un Aragon Jupiter de sa propre genèse. Ce faisant, il essaiera de suivre le mouvement de l'écriture d'un poème et de montrer comment des tâtonnements, des thèmes ou des métaphores inaboutis vont alimenter, bien longtemps après, l'écriture romanesque.

\section{Étude de cas : description du matériau génétique, transcriptions et hypothèses}

Pour cette incursion dans le domaine merveilleux de la genèse de l'écriture poétique d'Aragon, je ne prendrai qu'un seul exemple pour sa valeur emblématique. Il s'agit du poème « On dormait encore en ce temps-là 3 » du recueil de la poésie de la Résistance La Diane française qu' Aragon a composé en 1944 à la demande de son ami Pierre Seghers pour sa collection «Poésie 44 » à partir de poèmes prépubliés dans des revues ( «La rose et le réséda», fig. 1, «Le conscrit des cent villages», «Il n'y a pas d'amour heureux », «Elsa au miroir», etc.) et de six inédits, dont ce poème et le poème-préface «Ô mares sur la terre au soir de mon pays» (fig. 2 et 3). Le dossier génétique de La Diane française conservé au département des Manuscrits de la $\mathrm{BnF}$ contient pour l'essentiel des manuscrits de mise au net des vingt-cinq poèmes du recueil. Le seul poème à comporter des manuscrits de travail est précisément «On dormait encore en ce temps-là», qui apparaît ainsi comme un de

1. Cela aura été l'essentiel du travail pionnier de Renate Lance-Otterbein et des chercheurs du premier groupe de recherche Aragon-Triolet du CNRS autour de Michel Apel-Muller, Suzanne Ravis et Lionel Follet. 2. J'ai déjà étudié la genèse paradoxale d'Aurélien. Voir «La théorie des incipit à la lumière des manuscrits » dans M. Hilsum, C. Trévisan et M. Vassevière (dir.), Lire Aragon, actes du colloque du centenaire Aragon, Champion, 2000. Pour compléter ce travail, je montrerais que la genèse d'Aurélien ne peut se comprendre que comme une genèse croisée avec celle des Communistes. J'ai étudié aussi la genèse non moins complexe et non moins paradoxale de Théâtre/Roman dans «Autofiction et mentirvrai chez Aragon : les aveux de la génétique» (dir. J.-L. Jeannelle et C. Viollet, Génétique et Autofiction, Bruylant-Academia, «Au cœur des textes» $\left.n^{\circ} 7,2007\right)$ et dans «Le manuscrit de Théâtre/Roman : jeux et chemins du sens », (dir. M.-C. Mourier et R. Waller, Théâtre/Roman d'Aragon. Un singulier pluriel, Presses universitaires de Valenciennes, 2013) où j'accordais une grande importance au «Manuscrit Ristat». S'il me fallait aujourd'hui poursuivre sur ce point, je montrerais, par exemple avec le chapitre de desinit du roman, «L'avenir immédiat», que ce manuscrit partiel offert par Aragon à Jean Ristat est un vrai document de genèse car, antérieur au manuscrit conservé à la $\mathrm{BnF}$, il fonctionne comme une butte-témoin qui permet de mettre en évidence les strates de l'écriture qu'Aragon appelle les différents «états».

3. Louis Aragon, Euvres poétiques complètes, t. I, Paris, Gallimard, coll. «Bibliothèque de la Pléiade», 2007, p. 1006. 


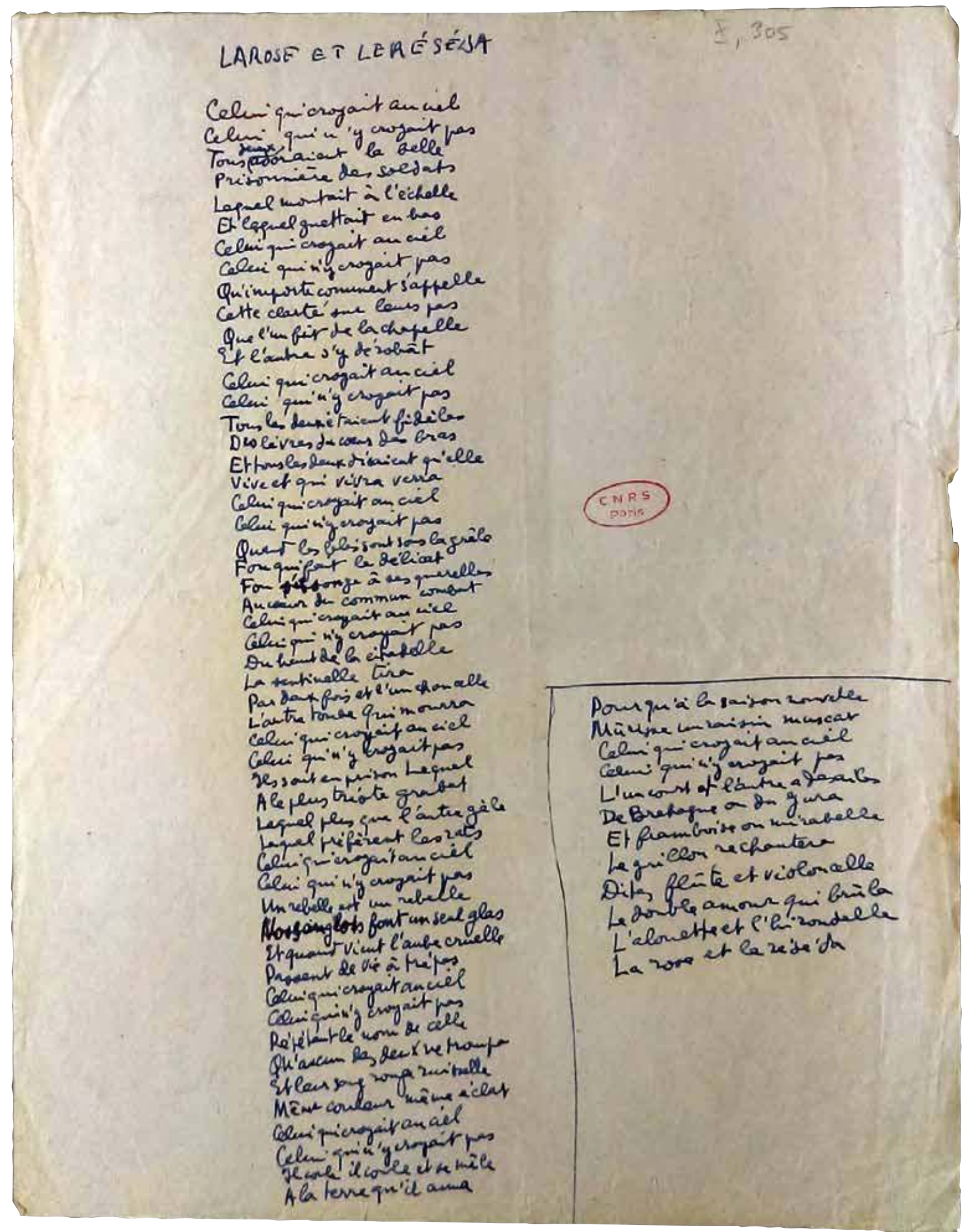

Fig. 1 : Mise au net de «La rose et le réséda».

(C) Paris, BnF, Fonds Aragon. Avec l'aimable autorisation de Jean Ristat 

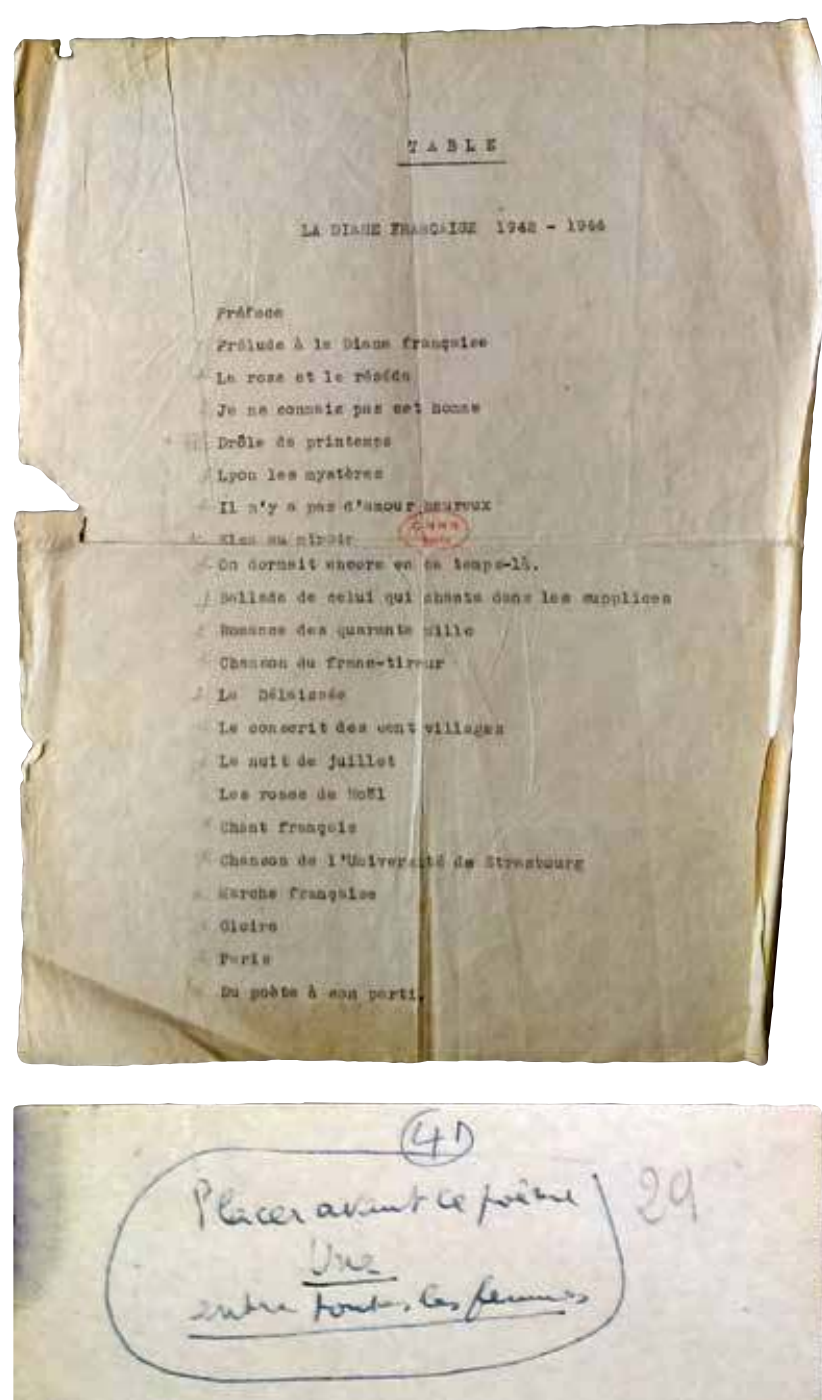

ONDORMAIT ENCORE EN CE TEMPS-L, $\mathcal{L} \quad / \bar{A}$

A

1. Ia fin jai trouvé dans la robe des anges Ce pli d'ombre et d'oubli doux comme un cachemire A la fin ce reposf ce port ou catte grange

Et le balancement d'une toile $y$ dérange

L'étaile vaguenent d'eau noire qui s'y mire

A la fia jai trouvé cetto prílte oú dornir

$$
\text { Anity }
$$

Ce vent de nufle part/ eette perte des sens

Mentrainent sur les pas diu parfum sans retour

Jenfonce dans le cour de ce bouquet d'absence

Je nais à cette huit d'une étrange naissance

Dans le pays défant qui m'ourre son velours

Oú rien ne serable plus se souvenir du jour

L'instant que met la pierre à foucher l'eau dormante De la mangelle d'ombre au reflet du soleil

Est assez long pour croire aux floraisons démentes
Fig. 2 : Table des matières des poèmes de La Diane française, publiée par Seghers.

(C) Paris, BnF, Fonds Aragon. Avec l'aimable autorisation de Jean Ristat

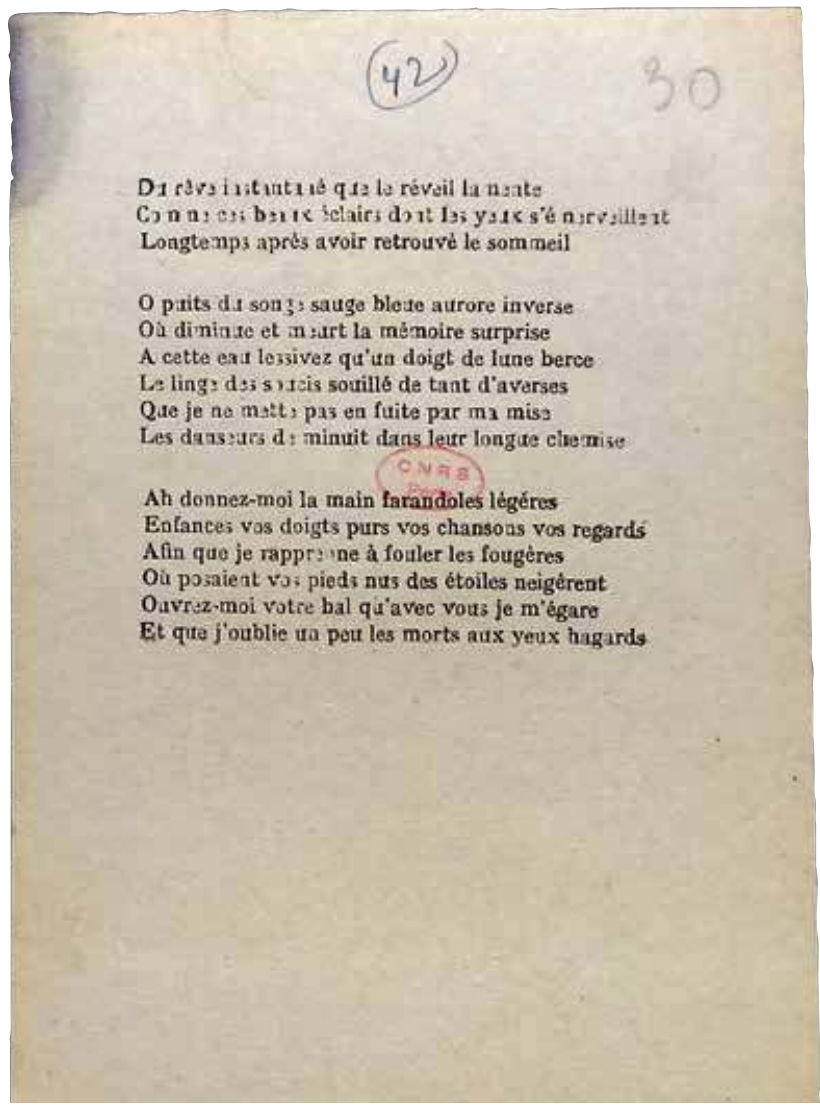

Fig. 3 : Page des épreuves de l'édition Seghers.

() Paris, BnF, Fonds Aragon. Avec l'aimable autorisation de Jean Ristat 


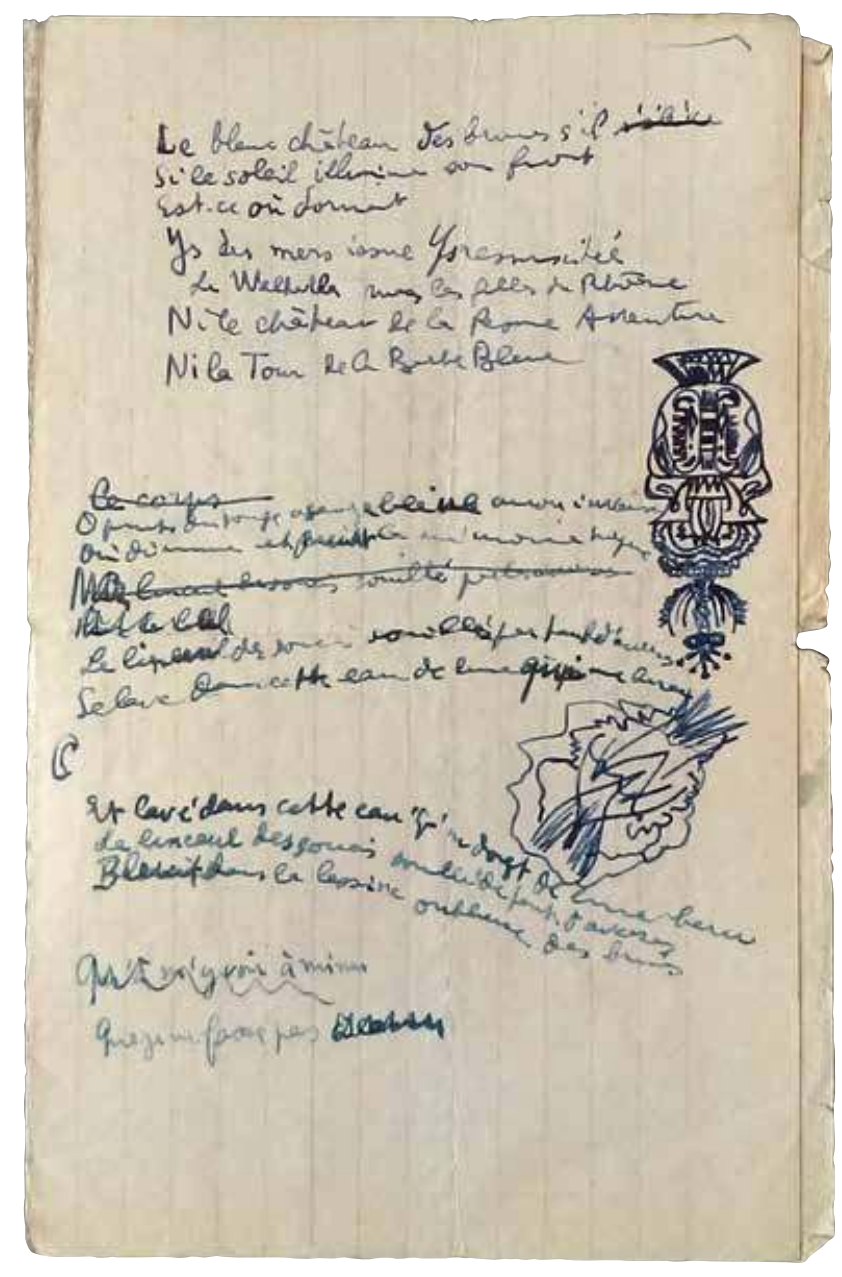

ces cailloux du petit Poucet qu'Aragon a laissés dans les archives du fonds Aragon-Elsa Triolet pour guider quelque chercheur de l'avenir...

Le dossier génétique de «On dormait encore en ce temps-là» contient deux feuillets d'avant-textes (fig. 4 à 8 ) et aucun manuscrit de mise au net complet : il s'agit d'un feuillet de papier à lettres avec interlignes grand format plié en deux puis en quatre (feuillet 1) et d'un feuillet de carnet sans interlignes (feuillet 2). Le recto du feuillet 1 comprend quatre zones écrites perpendiculairement aux interlignes (signe que ce feuillet a bien été plié en deux puis en quatre avant l'écriture) : au recto de la pliure en deux et en haut,

\section{Feuillet $1 r^{\circ}$. État $1-a$}

Le blanc château des brumes s'il s'élève

Si le soleil illumine son front

Est-ce où dormait

Ys des mers issue Ys ressuscitée

Le Walhalla nues les filles du Rhône

Ni le château de la Pesme Aventure

$\mathrm{Ni}$ la Tour de la Barbe Bleue

\section{Feuillet $1 r^{\circ}$. État $1-b$}

Ce corps

Ô puits du songe ô sauge bleue aurore inverse Où diminue *et geint* la mémoire surprise

Mon linceul de soucis souillé par les averses *Par le bat*

Le linceul des soucis souillé par tant d'averses

Se lave dans cette eau de lune qui me berce

Et lavé dans cette eau qu'un doigt de lune berce Le linceul des soucis souillé par tant d'averses Bleuit dans la lessive oublieuse des brumes

Qu'à m'y voir à min

Que je ne fasse pas [illis.]

Fig. 4 : «On dormait encore en ce temps-là», feuillet $1 \mathrm{r}^{\circ}$, quart haut droit.

( Paris, BnF, Fonds Aragon. Avec l'aimable autorisation de Jean Ristat

une ébauche d'une strophe qui ne sera pas retenue (état 1-a); en bas, au-dessous de la pliure en quatre, une ébauche de ce qui sera l'avant-dernière strophe, la strophe 4 (état 1-b); au verso de la pliure en deux et en haut, une autre ébauche de la strophe 4 (état 1-c); en bas, au-dessous de la pliure en quatre, l'ébauche d'un vers qui ne sera pas conservé : «Mais lorsque j'ai touché l'oubli couleur» (état 1-d). Quant au verso de ce feuillet, qui est l'intérieur de la page pliée, il se lit donc déplié et contient la première ébauche du poème, sans titre, mais avec le même incipit, «À la fin j'ai trouvé» et les quatre premières strophes (état 2). La feuille de carnet écrite à l'encre turquoise contient au recto une 


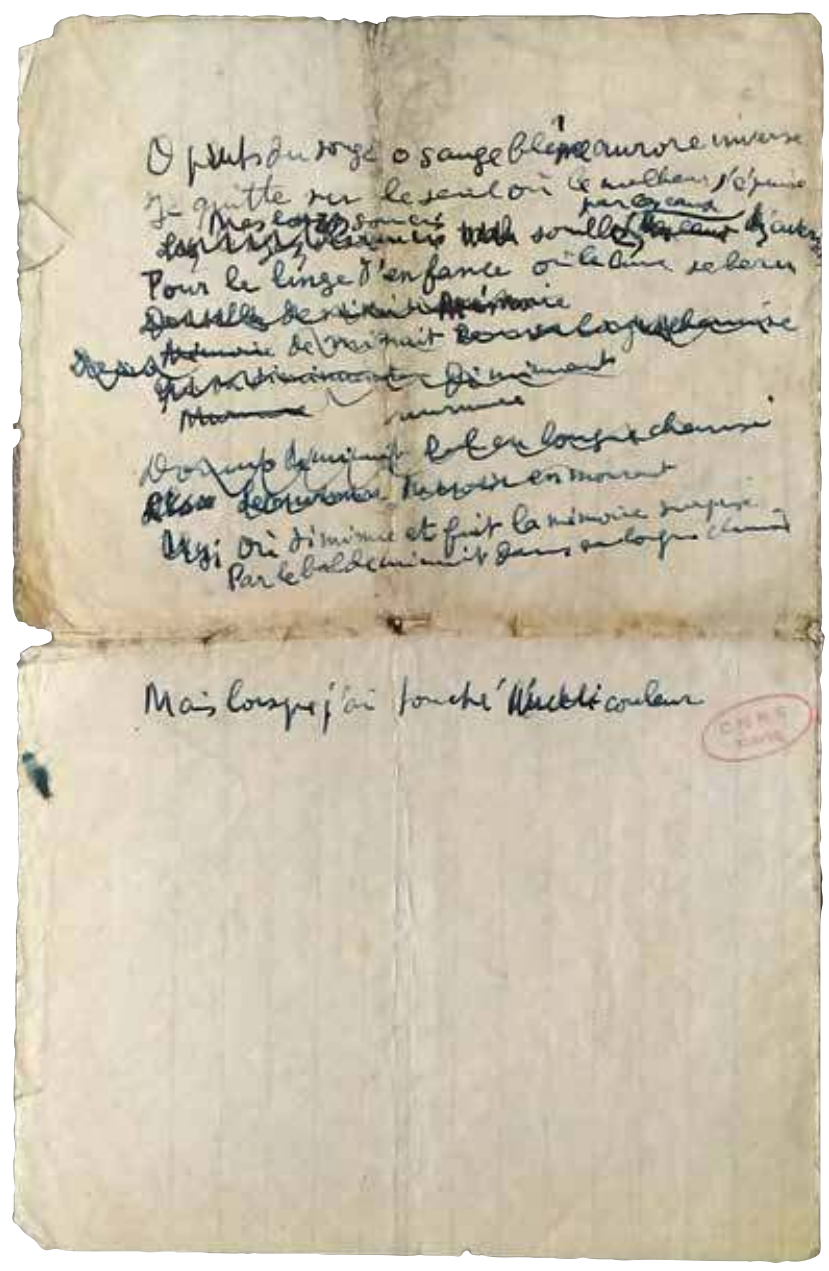

nouvelle ébauche des quatre premières strophes (état 3 ) et au verso diverses ébauches à l'encre bleu turquoise de la dernière strophe (strophe 5 ) et en bas, au crayon, une mise au net de cette strophe 5 (état 4).

Je fais l'hypothèse que la page pliée en deux, puis en quatre (peut-être pour la mettre dans une poche et la transporter plus facilement...) constitue l'état le plus ancien du texte : des demi-pages suffisent pour des ébauches, des brouillons, des gammes, pour la recherche des strophes et la rêverie avant que le poème prenne forme, ce dont témoignent deux dessins griffonnés en marge comme pour attendre l'inspiration. Tandis que la pleine page est

\section{Feuillet $1 r^{\circ}$. État 1-c}

Ô puits du songe ô sauge blême aurore inverse Je quitte sur le seuil où le malheur s'épuise Mes longs soucis par les eaux Le linceul des soucis souillé des eaux des averses Pour le linge d'enfance où la lune se berce Dormeurs de minuit *de* mémoire

Mémoire de minuit dans sa longue chemise

Qui va diminuant diminuant Murmure murmure Dormeurs de minuit bal en longue chemise Elle/*Elsa* Le murmure du monde en mourant Hy/*Lui* Où diminue et fuit la mémoire surprise Par le bal de minuit dans sa longue chemise

4 Feuillet $1 r^{\circ}$. État 1-d

Mais lorsque j'ai touché l'oubli couleur

Fig. 5 : «On dormait encore en ce temps-là », feuillet $1 \mathrm{r}^{\circ}$, gauche. (C) Paris, BnF, Fonds Aragon. Avec l'aimable autorisation de Jean Ristat

nécessaire à l'écriture du poème : le poète ouvre la page des ébauches pliée en deux et commence le poème «À la fin j'ai trouvé ». Mais sur cette première mise au net, la fin s'écrit encore dans les tâtonnements. Le poète refait alors une nouvelle mise au net sur le recto de la feuille du carnet où il manque encore la strophe 5 et il utilise le verso pour écrire l'ébauche de cette dernière strophe avant d'en noter au crayon la mise au net. Il suffira donc d'un nouveau feuillet pour noter la mise au net définitive de tout le poème, mais ce feuillet est absent du dossier. Et le tapuscrit définitif aussi.

Je propose donc la transcription diplomatique de ces différentes zones des feuillets; elles correspondent à 


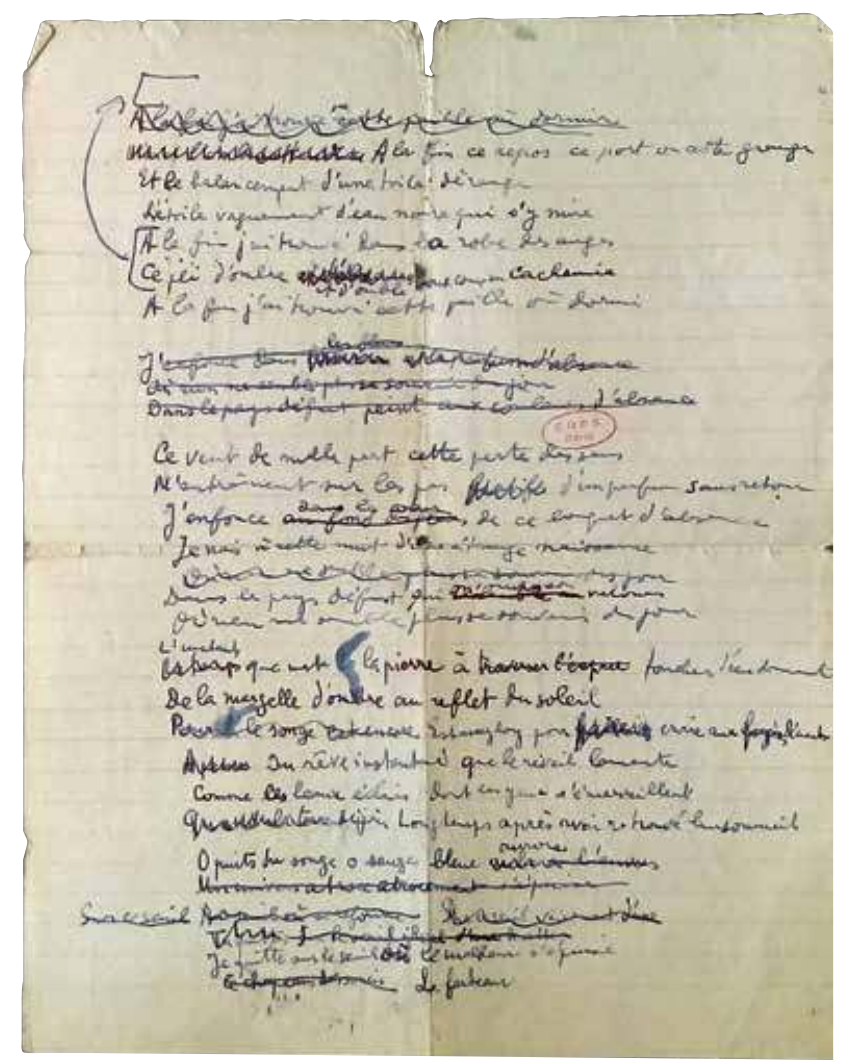

Fig. 6 : «On dormait encore en ce temps-là», feuillet $1 \mathrm{v}^{\circ}$, page dépliée. (C) Paris, BnF, Fonds Aragon. Avec l'aimable autorisation de Jean Ristat
4 Feuillet $1 v^{\circ}$. Page dépliée. État 2

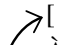

À la fin j'ai trouvé cette paille où dormir

[Mots barrés illis.] À la fin ce repos ce port ou cette grange

Et le balancement d'une toile dérange

L'étoile vaguement d'eau noire qui s'y mire

À la fin j'ai trouvé dans la robe des anges

Ce pli d'ombre [mots barrés illis.] et d'oubli doux comme un cachemire

À la fin j'ai trouvé cette paille où dormir

$$
\text { les fleurs }
$$

J'enfonce dans [illis. ] et le parfum d'absence

Où rien ne semble plus se souvenir du jour

Dans le pays défunt peint aux couleurs d'absence

Ce vent de nulle part cette perte des sens

M'entraînent sur les pas furtifs d'un parfum sans retour

[Strophe 2]

J'enfonce [au fond des fleurs] de ce bouquet d'absence

Je nais à cette nuit d'une étrange naissance

Où rien ne semble plus se souvenir des jours

Dans le pays défunt qui m'ouvremble au velours

Où rien ne semble plus se souvenir du jour

L'instant

Le temps que met la pierre à traverser l'espace toucher l'eau dormante

De la margelle d'ombre au reflet du soleil

Pourlesonge estencore Est assez long pour [illis.] croire aux fougères démentes

*À peine* Du rêve instantané que le réveil lamente

Comme ces beaux éclairs dont les yeux s'émerveillent

Quand la terre déjà Longtemps après avoir retrouvé leur sommeil

aurore

Ô puits du songe ô sauge bleue aube à l'envers

[Strophe 4] Un univers atroce atrocement s'épuise

Sur ce seuil Au seuil où se reforme Sur ce seuil vainement d'une

Tu quittes sur le seuil idéal d'une de cette

Je quitte sur le seuil où le malheur s'épuise

Le Chapeau des soucis Le fardeau 


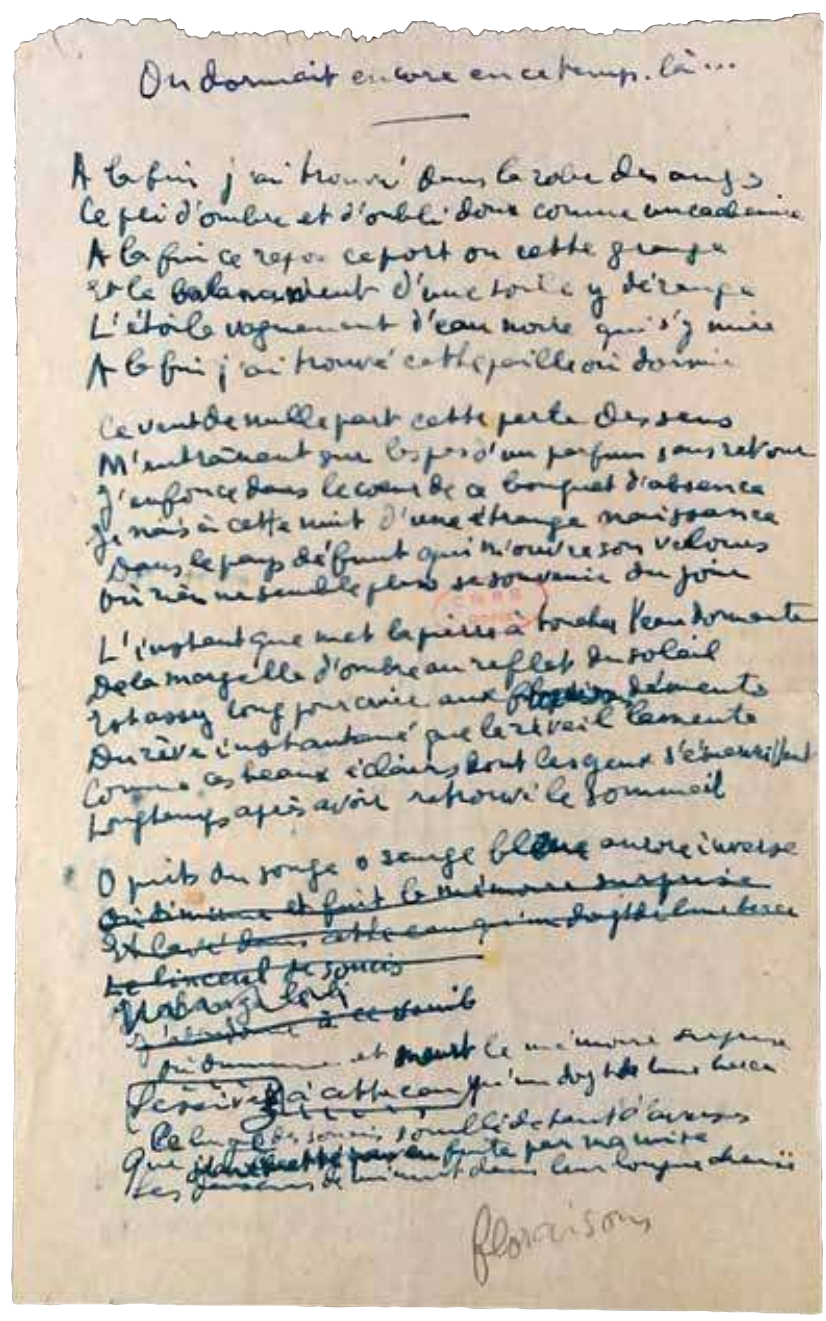

Fig. $7:$ : On dormait encore en ce temps-là », feuillet $2 \mathrm{r}^{\circ}$.

(๑) Paris, BnF, Fonds Aragon. Avec l'aimable autorisation de Jean Ristat

\section{Feuillet $2 r^{\circ}$. État 3}

On dormait encore en ce temps-là...

À la fin j'ai trouvé dans la robe des anges Ce pli d'ombre et d'oubli doux comme un cachemire À la fin ce repos ce port ou cette grange Et le balancement d'une toile y dérange L'étoile vaguement d'eau noire qui s'y mire À la fin j'ai trouvé cette paille où dormir

Ce vent de nulle part cette perte des sens M'entraînent sur les pas d'un parfum sans retour J'enfonce dans le cœur de ce bouquet d'absence Je nais à cette nuit d'une étrange naissance Dans le pays défunt qui m'ouvre son velours Où rien ne semble plus se souvenir du jour

L'instant que met la pierre à toucher l'eau dormante De la margelle d'ombre au reflet du soleil

Est assez long pour croire aux fougères/floraisons démentes Du rêve instantané que le réveil lamente Comme ces beaux éclairs dont les yeux s'émerveillent Longtemps après avoir retrouvé le sommeil

Ô puits du songe ô sauge bleue aurore inverse Où diminue et fuit la mémoire surprise Et lavé dans cette eau qu'un doigt de lune beree Le linceul des soucis

Je change le bi

J'abandonne à ce setuil

Où diminue et meurt la mémoire surprise Lessivée/ez à cette eau qu'un doigt de lune berce Le linge des soucis souillé de tant d'averses Que je ne n'aille/mette pas en fuite par ma mise Les danseurs de minuit dans leur longue chemise

\section{floraisons}




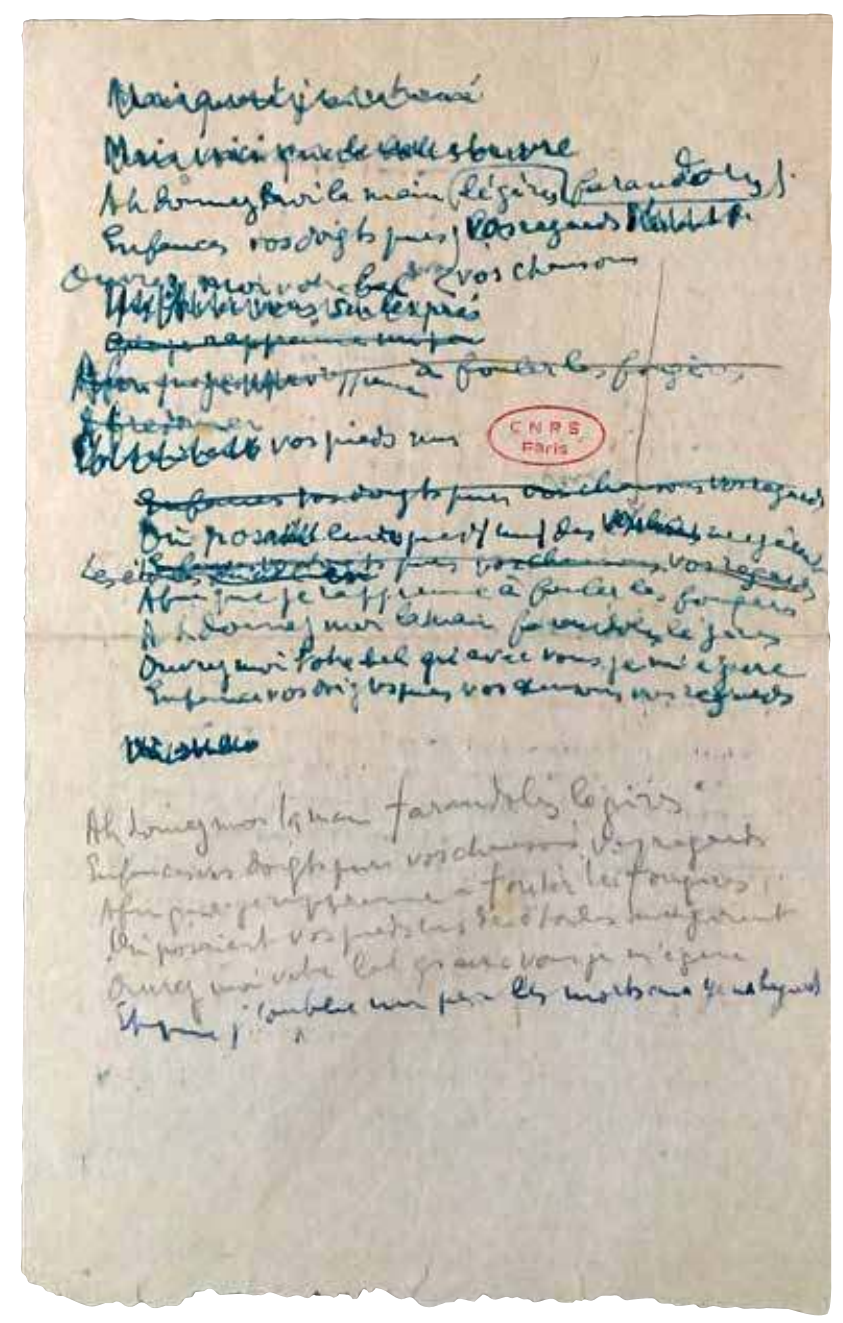

différentes étapes de l'écriture, à différents «états » du texte, qui sont comme des strates où se sédimente petit à petit le poème en cours d'écriture.

\section{Le mouvement de la genèse}

Ces rares documents de genèse de la poésie de la Résistance d'Aragon, comme rescapés des temps difficiles de l'Occupation et des nombreux déplacements du couple

\section{Feuillet $2 v^{\circ}$. État 4}

Mais quand j'ai treuvé

[Strophe 5]

Mais voici que le voile s'ouvre

Ah donnez moi la main/légèresfarandoles

Enfances vos doigts purs /vos regards *étoile*

Ouvrez-moi votre bal vos chansons

Qu'[illis.] vous sur les prés

Que je rapprenne un peu

Afin que je [illis.] rapprenne à fouler les fougères

À fredonner

En stivant vos pieds nus

Enfances vos doigts purs vos chansend vos regards

Où posaient leurs pieds nus des vers luisants *surgirent*

Enfances vos doigts purs vos chansons, vos regards,

tes étoiles du eiel en

Afin que je rapprenne à fouler les fougères

Ah donnez moi la main farandoles légères

Ouvrez-moi votre bal qu'avec vous je m'égare

Enfance vos doigts purs vos chansons vos regards

[illis.]

Ah donnez-moi la main farandoles légères

Enfances vos doigts purs vos chansons vos regards

Afin que je rapprenne à fouler les fougères

Où posaient vos pieds nus des étoiles neigèrent

Ouvrez-moi votre bal qu'avec vous je m'égare

Et que j'oublie un peu les morts aux yeux hagards

Fig. 8 : «On dormait encore en ce temps-là », feuillet $2 v^{\circ}$.

(C) Paris, BnF, Fonds Aragon. Avec l'aimable autorisation de Jean Ristat

en zone Sud, semblent comme une butte-témoin de la lente sédimentation du texte poétique. Les quatre zones écrites au recto du feuillet 1 contiennent les premiers tâtonnements sur le thème de la mémoire et de l'oubli qui sera le thème majeur de ce poème, et peut-être la tentation profonde du poète en contrepoint à la poésie militante de la guerre. Deux signes majeurs de ce statut d'avant-texte : l'absence de versification de la strophe de sept vers (ce n'est pas encore le sizain qui sera adopté ensuite...) sans rimes (ressuscitée/ Rhône/Aventure/bleue) dans l'état 1-a et les dessins à la 
plume griffonnés en marge de l'état 1-a et de l'état 1-b. Alors le poète rature, fait des pâtés qui alimenteront ensuite le redémarrage de l'écriture, peut-être quelques heures après : ainsi la tache d'encre en forme de demi-lune est peut-être ce qui amorce le vers suivant : «Et lavé dans cette eau qu'un doigt de lune berce» de l'état 1-b, lui-même - ainsi que les trois autres vers de la fin de la strophe - écrit en biais parce que le bas du dessin gribouillé empêche la fin du vers de suivre la ligne.

Après ces premières ébauches sur des mots-thèmes ( «Le blanc château des brumes » de l'état 1-a et le «puits des songes » de l'état 1 -b et de l'état $1-c)$, le poème ne peut advenir qu'avec l'apparition du sujet lyrique. C'est ce qui se fait avec le vers unique «Mais lorsque j'ai touché l'oubli couleur» de l'état 1-c, aussitôt abandonné pour passer à la pleine page du feuillet plié en quatre et maintenant déplié. Ici commence l'état 2.

Après de nouvelles ratures et déplacements, le sujet s'affirme dans un vers, qui, de cette manière métalinguistique si chère à Aragon, mime presque les recherches infructueuses des demi-pages précédentes : «À la fin j’ai trouvé cette paille où dormir », qui donne le thème principal (le sommeil) et le titre à venir, qui ne sera trouvé qu'à l'étape suivante. Et cet incipit de la strophe 1 trouvé, qui donne aussi le mètre (l'alexandrin), le branle est donné au poème qui sera fait de sizains d'alexandrins à rimes croisées. L'apparition de la versification est donc concomitante à celle du sujet lyrique, qui balance parfois entre le je et le tu, par une sorte d'apostrophe à soi-même ( «Tu quittes le seuil idéal») qui ne sera pas conservée dans l'avancée de l'écriture.

Cet état 2 se poursuit dans de nouveaux tâtonnements qui ont maintenant le cadre fixé du mètre et de la strophe, mais qui portent désormais sur la place des mots dans le vers. Ce sont d'infinis ratures et retours comme des hésitations de la voix, des balbutiements : ainsi les variations sur la place du «seuil» à la fin de l'état 2 («Sur ce seuil», «Au seuil», «Sur ce seuil vainement», «Tu quittes sur le seuil», «Je quitte sur le seuil»). Ou encore les multiples déplacements des couples noms/adjectifs ou verbes/ compléments - matérialisés par le signe typographique de l'inversion des mots que la transcription diplomatique ne peut pas rendre - de l'état 3 : «légères farandoles »/ «farandoles légères », «lessivez à cette eau » / «à cette eau lessivez».
Avec les deux feuillets manuscrits de ce poème de la Résistance, on voit comment petit à petit les fragments s'agglomèrent et comment ce qui était initial (un vers, une strophe) se trouve renvoyé à la fin, après insertion de nombreux développements ${ }^{4}$. Ainsi, par exemple, la première rêverie du poète sur le «puits du songe» (état $1-b$, état $1-c$ et même état 2 et état 3 ) ne constituera que la strophe 4 du poème. De même, le vers «Enfances vos doigts purs vos chansons vos regards » de la strophe 5 connaît de nombreux déplacements dans l'état 4 : il est renvoyé du début à la fin de la strophe, pour finalement se retrouver au début de la dernière strophe et rimer avec un vers ajouté in extremis à l'encre bleue sur la mise au net au crayon de cette strophe finale : «Et que j'oublie un peu les morts aux yeux hagards ». Le mouvement perpétuel de l'écriture se produit ainsi autour de noyaux ou de foyers initiaux, qui en quelque sorte «donnent le la», selon l'expression qu'Aragon emploie à propos d'Aurélien dans Je n'ai jamais appris à écrire ou les incipit ${ }^{5}$, puis qui se trouvent agglomérés à un autre matériau textuel ultérieur et parfois même déplacés matériellement, en tout cas recontextualisés par les nouveaux apports de l'écriture : par exemple, la connotation du Grand Meaulnes de l'état 1-a («Le blanc château des brumes »), encore conservée dans l'état 1-c avec l'image du bal enfantin, va se trouver modifiée dans la version finale, et cela dès l'état 3 , par l'image des dormeurs contenue dans le titre. Cette métaphore des dormeurs et des chambres va d'ailleurs devenir dans l'écriture romanesque ultérieure, comme on le verra, un foyer d'inspiration incandescent, comme dans la nouvelle «Murmure » insérée dans La Mise à mort (1965) ou dans la nouvelle «Le contraire-dit» du recueil Le Mentir-vrai (1974) qui constitue une sorte de réécriture de «Murmure» avec le cadre de la chambre du couple, du lit et des rêves de la femme-enfant où se réécrit en se prolongeant dans l'enfance de «la petite e.»-le conte où rêvent la Murmure de La Mise à mort et son écrivain de mari (cf. encadré ci-dessous). D'ailleurs l'état 1-c qui donne forme à l'image des «dormeurs de minuit» - elle disparaitra dans l'état 2 pour subsister sous la forme des «danseurs de

4. C'est aussi le cas pour l'écriture des romans. Voir par exemple la genèse des Communistes.

5. ORCr, t. XLII, p. 239. 
minuit »-, contient, en marge de ces vers qui introduisent la référence au Grand Meaulnes, deux gribouillis où surnagent en surimpression à deux mots illisibles raturés les mots qui vont alimenter l'écriture romanesque ultérieure d'inspiration autobiographique : «Elsa» et «Lui » ou «Louis».

\section{NOYAU ET INCIPIT : GENÈSE DE LA NOUVELLE «LE CONTRAIRE-DIT» DU MENTIR-VRAI}

Noyau initial et matrice, l'exemple de «Murmure» dans La Mise à mort comme «Le contraire-dit» relèvent d'ailleurs de cette même règle de l'avancée de l'écriture à partir d'un noyau initial - et c'est peut-être cela après tout qu'Aragon appelle un incipit... C'est ainsi que la nouvelle «Murmure» semble être le véritable avant-texte de La Mise à mort autour duquel tout le roman va s'organiser. De la même manière la nouvelle «Le contraire-dit» procède aussi d'une écriture en deux temps ou de deux «campagnes d'écriture ». Pré-publiée dans Les Lettres françaises $\mathrm{n}^{\circ} 1427$ du 15 mars 1972, deux ans après la mort d'Elsa le 16 juin 1970, elle se construit à partir d'un noyau ancien écrit avant la mort d'Elsa et dont témoigne une lettre à Lili Brik du 5 mars 1969 : «Aragon a justement écrit un récit, le plus tendre et le plus doux - c'est ce qu'il dit lui-même sur le petit e (c'est-à-dire en russe "è”) de rêve en rêve ${ }^{1}$.» Ce sont des ajouts selon la technique du couper-coller et non plus de la «paperolle» proustienne (Aragon découpe les anciens feuillets et y intègre de nouveaux développements en les collant avec du scotch dans le sens de la page qui se trouve alors dépasser le format A4 et « dénuder ainsi le procédé»...) et quelques ratures capitales à l'incipit de la nouvelle («juin», «juins» barrés et remplacés par «été » 2 , «étés » comme pour exorciser ce mois de la mort d'Elsa...) qui vont reconfigurer le texte ancien. C'est ainsi que la mort d'Elsa, «la petite e » de la nouvelle, se trouve inscrite dans le texte et redonne un sens poignant à certaines images du texte ancien : «et le doute surprend la gorge, de ce qu'il est advenu d'e., l'a-t-on, comme une feuille, entraînée parmi les pailles, les poussières, les venelles, sans nom, les granges de la mémoire, les haltes d'autobus à l'aube, les chemins de fer dans la nuit ${ }^{3}$ ? » Et ce phénomène de la recontextualisation du texte primitif produit par les ajouts et les collages probablement postérieurs à la mort d'Elsa donne au contenu de la nouvelle et à son titre un sens nouveau : un troisième sens qui vient s'ajouter aux deux sens premiers déjà contenus dans le texte de la nouvelle dont le manuscrit aura été lu par Elsa elle-même qui en aura été la première lectrice comme en témoigne sa lettre à Lili. Le premier sens du titre mystérieux est lié à la problématique amoureuse et permet l'aveu sur le «mur de verre des mots » 4 . Dans cette quête de la femme perdue, cette métaphore du contraire-dit (prendre toujours le sens inverse pour espérer peut-être rencontrer la petite e...) suggère qu'il n'y a plus de lieux pour être ensemble et qu'il faut imaginer des ruses de Sioux pour se retrouver, pour communiquer. Le deuxième sens du titre est la dénégation historique, liée à la douleur de la faute historique de l'URSS en Tchécoslovaquie (la nouvelle se termine par le collage du communiqué de l'Agence Tass du 21 août 1968 à la radio). À ces deux sens s'en ajoute maintenant un troisième : dire l'absence définitive d'Elsa qui inverse toutes les significations des choses, comme en témoigne un fragment ajouté, scotché sur le folio 3 , où le monologue intérieur passe du féminin (la petite fille puis la femme qui rêve) au masculin (son mari et celui qui écrit) : "Qu'est-ce qui est le rêve et qu'est-ce qui est le contraire du rêve? On croit facile de répondre à cette question, quand on n'y a pas réfléchi. Par exemple, on dira : le rêve, c'est le contraire de la vie. Comme si les morts rêvaient ! [...] Quelque chose est le contraire du rêve dont le rêve est le contraire, mais quoi ? [...] Il s'est passé quelque chose de terrible. Et je ne sais plus quoi ${ }^{5}$.»

1. Léon Robel (dir.), Correspondance Lili Brik-Elsa Triolet 1921-1970, Paris, Gallimard, 2000, p. 1476.

2. Louis Aragon, CEuvres romanesques complètes (ORC), t. V, p. 871.

3. Ibid., p. 881.

4. Louis Aragon, Blanche ou l'oubli, dans $O R C, \mathrm{t}$. V, p. 701.

5. Louis Aragon, $O R C$, t. V, p. 874.

Enfin ces nombreuses ratures en cours d'écriture produisent souvent d'heureuses avancées ou des approfondissements du sens par la bifurcation des métaphores. Par exemple, une rature transforme le «linceul des soucis » (état 1-b) en « linge des soucis » (état 3), effaçant la connotation mortifère et introduisant la connotation mélancolique de l'enfance ( «le linge d'une enfance où la lune se berce» dans l'état 1-c) et la référence explicite au souvenir du bal des enfants dans Le Grand Meaulnes («Par le bal de minuit dans sa longue chemise» dans l'état 1-c), qui sera finalement effacée - ou seulement implicite - dans la version finale : «Les danseurs de minuit dans leur longue chemise» (état 3 ). 
Ainsi progressivement, dans cette rêverie très intime que constitue ce poème assez à part dans la production poétique de la guerre, le goût de vivre l'emporte sur le pessimisme et la nostalgie de l'enfance comme du songe surréaliste emblématisé par la «sauge bleue ${ }^{6}$ » - celle-là même qui alimentera le désir de faire revivre les années vingt de la jeunesse surréaliste d'Aragon dans l'écriture d'Aurélien en plein cœur de la guerre - apparaît comme le contrepoint et le remède aux malheurs d'un «temps misanthrope».

C'est ici que, pour éclairer la genèse de ce poème, il faut recourir à une autre sorte d'avant-texte : la correspondance d'Aragon avec ses amis américains, Hanna et Mattew Josephson 7 . Dans une lettre émouvante du 5 septembre 1940, Aragon raconte sa guerre, comme il le refera sur le mode romanesque en se lançant dans le récit de maijuin 1940 dans Les Communistes :

En quelques mots voici ce qui s'est passé : je me suis trouvé en tête des premières troupes françaises entrées en Belgique et j'ai fait toute la campagne des Flandres dans des conditions extrêmement aventureuses. [...] Cela a été une guerre rapide et romanesque pour moi, mais dont les conséquences sont plus terribles pour le pays que vous ne l'imaginez même. [...] Remarquez que tout ce qui précède me trahirait, si vous en déduisiez que je suis devenu pessimiste ${ }^{8}$.

Et le 22 octobre, il répond par la négative à une proposition de Mattew Josephson et Malcom Cowley d'écrire des articles «alimentaires» dans des revues américaines comme la New Republic sur les écrivains français dans la France occupée. Il explique très humainement et très honnêtement pourquoi il ne lui est pas possible d'être le journaliste qui raconterait cette misère dans «le pays défunt» qui est le sien, comme le dit un vers de «On dormait toujours en ce temps-là » :

Il est à peu près impossible pour moi (ce n'est pas une affaire de censure) de parler des difficultés quotidiennes des écrivains, des autres écrivains, premièrement parce que je ne les connais pas, parce qu'il n'y a plus en général entre eux et moi quoi que ce soit qui me permette de le faire, et que ce qui leur est arrivé, je l'ignore. D'une façon générale, je suis sensiblement obligé de m'interdire «le journalisme» parce que dire les choses comme elles sont, pour un journal étranger (ou une revue), dans la mesure où je les connais, serait très mal interprété, et que je ne sais pas leur donner un aspect faux à la fois et intéressant pour le public américain. Une sorte de pudeur nationale m'interdirait de parler de certains cas, et par suite ne laisserait que les banalités dont vous n'avez pas besoin. Pour être plus clair, nous sommes des gens plongés dans notre défaite, et rien ne me paraît digne d'être dit qui ne tienne compte de cette défaite, et de mes sentiments à ce sujet; mais alors cela devient tout à fait impossible.

Cette douleur du «pays défunt», qui s'exprime ici dans la familiarité et la simplicité d'une lettre à des amis, donne peut-être un indice sur la datation de ces feuillets anciens du poème dont on ne sait pas exactement quand il a été écrit sous l'Occupation. Pierre Seghers demande à Aragon de réunir des poèmes pour son édition de «Poésie 44 », Aragon y rassemble des poèmes déjà publiés et aussi des inédits qui n'ont pas trouvé leur place dans d'autres recueils ou qui ont été écrits dans ces derniers mois de la guerre. C'est peut-être le cas de ce poème «On dormait encore en ce temps-là » mais peut-être les premiers états en sont-ils plus anciens et contemporains de ces lettres aux Josephson au début de l'Occupation, au moment où le souvenir de la campagne de France et de la défaite de mai-juin 1940 est encore vif, où la tentation de l'oubli est tout à fait compréhensible et où s'inaugure la métaphore de la femme-pays qui alimentera toute la poésie de la Résistance à venir. C'est une micro-rature en cours d'écriture de l'état 2 qui permet cette hypothèse : la proposition relative «qui ressemble au velours » pour caractériser «le pays défunt» devient «qui m'ouvre son velours », introduisant ainsi non seulement le sujet lyrique mais aussi la connotation amoureuse et la superposition de la France et de la femme aimée. Un des thèmes majeurs de la poésie de la Résistance d'Aragon trouve peut-être ici son origine. Ainsi que le choix de l'intertexte médiéval qui irriguera tout le «trobar clus» de la poésie de contrebande : «Ys ressuscitée», «le Walhalla» et surtout «le château de la Pesme Aventure» dans Yvain ou le chevalier au lion de Chrétien de Troyes, trois références

6. Plante elle-même emblématique de l'imaginaire surréaliste d'Aragon : «La métalepse est de règle où la sauge fleurit» lit-on dans le Traité $d u$ style de 1928 (Gallimard, coll. «L'Imaginaire», p. 173).

7. Conservée à la Beinecke Library de l'université de Yale, elle a été découverte par Daniel Bougnoux qui en a rapporté une photocopie au fonds Aragon. Il en a donné aussi des extraits, en annexe des Communistes, dans le tome III de la Pléiade.

8. Louis Aragon, $O R C$, t. III, Paris, Gallimard, coll. «Bibliothèque de la Pléiade », 2003, p. 1317. 
littéraires qui constituent la base du premier sizain dans l'état 1-a. Et si elles disparaîtront des états ultérieurs du poème, elles réapparaîtront dans bien des poèmes écrits par Aragon sous l'Occupation, comme par exemple dans Brocéliande.

\section{Les restes : de la poésie au roman}

Ce très beau manuscrit du poème de La Diane française peut être considéré comme une sorte d'avant-texte des figures de Murmure et Fougère dans La Mise à mort, mais aussi de Blanche ou l'oubli pour la problématique de la mémoire et même de Théâtre/Roman pour la thématique du secret sur les camps au chapitre «Daniel ou le metteur en scène». En effet, on trouve ici une première réflexion sur l'oubli liée à la guerre et aussi un recours au rêve contre la misère du présent (la lessive du linge des soucis dans le «puits du songe »). Cela résonne comme un premier appel au passé surréaliste, une nostalgie de son retour. L'oubli est associé au retour vers l'enfance (les «farandoles légères ») de même qu'on trouvera aussi la thématique de l'enfance de Daniel dans Théâtre/Roman et l'oubli de l'humiliation des camps nazis. Ce manuscrit témoigne en outre des hésitations de l'écriture avec la rature de la mythologie : la référence à Ys, la ville endormie est supprimée. Il s'agit là d'une très grande hésitation : elle maintient un suspens qui va produire le roman bien longtemps après, dans les années soixante, loin de l'expérience de l'Occupation... Et de ces reliquats, certains, comme ce vers «Un univers atroce atrocement s'épuise », renvoient à d'autres mots déjà utilisés pour dire l'Occupation dans les nombreux recueils poétiques de la guerre, comme ce «temps misanthrope» des Yeux d'Elsa. Et d'autres, comme les dessins qui accompagnent cette ébauche, renvoient à une pratique de la période surréaliste dont témoignent certaines lettres d'Aragon à Breton maintenant éditées et à une pratique qui sera reprise et amplifiée sans honte par le vieux poète dans ses dernières années. Si bien que ces magnifiques ébauches du poème de La Diane française nous rendent sensible le fait que rien ne se perd dans la création littéraire chez Aragon et que tout se transforme, comme si les balbutiements de l'écriture, qui sont aussi ceux de l'imagination et de la mémoire, portaient à l'existence un stock de matériaux bruts - stock de mots et d'images - que le temps va faire ressurgir et porter à maturité quand le moment sera venu, signe que l'écriture poétique et l'écriture romanesque s'épanouissent sur le même terreau.

On voit ainsi comment ce manuscrit prend tout son sens d'être situé dans le contexte de la guerre où il a été produit mais s'éclaire aussi à rebours d'être confronté à toute l'œuvre romanesque de la dernière carrière d'Aragon qu'il éclaire à son tour. On trouve ainsi à travers les métaphores balbutiantes le paradis perdu de l'enfance à retrouver et où «s'égarer» pour oublier les horreurs du présent brutalement apparues dans le dernier vers («les morts aux yeux hagards »). Cette thématique de la mémoire, un temps convoquée pour faire face aux douleurs de l'Occupation, va se trouver ensuite évacuée (avec ce détail très symptomatique du mot «oubli» dans le vers «oublié» de l'état 1-d) au profit des thématiques combattantes de la poésie de la Résistance. Mais cette présence si forte de la mémoire va ressurgir d'abord dans l'écriture romanesque parallèle en ce temps de la guerre (l'écriture d'Aurélien évoquée plus haut où vont ressurgir les années 1920, ce temps de la jeunesse surréaliste d'Aragon), pour s'épanouir enfin dans la dernière carrière romanesque d'Aragon : les fougères et les murmures qui sont ici ceux du temps perdu de l'enfance vont devenir les noms mêmes (Fougère et Murmure) de la femme aimée par Alfred et Anthoine dans La Mise à mort. De même, la référence au Grand Meaulnes, raturée ou du moins estompée comme on l'a vu plus haut, va devenir un intertexte exhibé dans Blanche ou l'oubli.

On voit aussi comment la germination des métaphores (le puits du songe, la lessive bleue de la mémoire, les «fougères du trouble» dans un autre poème de La Diane française, «Lyon-les-mystères », les murmures) s'enracine dans les circonstances de la guerre pour en être à la fois l'affirmation (les «fougères de l'ombre» pour dire «l'armée de l'ombre» de la Résistance) et la dénégation (les «fougères de l'ombre» et les murmures pour dire le rêve comme oubli du réel menaçant de la guerre). Ainsi ces métaphores balbutiées et pour certaines raturées de la poésie de la Résistance constituent à la fois l'instrument d'une poétique de la contrebande en même temps qu'une distance prise avec le pur engagement de la poésie. Ces métaphores dans la pure filiation de l'écriture surréaliste (comme « cette paille où dormir » ou «les floraisons démentes du rêve») 
qui ressurgissent pendant l'Occupation, au moment du plus grand danger d'écrire et de vivre, annoncent les grandes interrogations des derniers romans après le tournant de 1956.

Ainsi se trouve annoncé et confirmé que pour Aragon la poésie est toujours «l'envers du temps», comme il le formule dans la première postface à L'Euvre poétique dans les années 1970, au moment même où il le montre aussi dans l'écriture romanesque de Blanche ou l'oubli et de Théâtre/Roman.

$*$

Si les dossiers génétiques des poèmes de la Résistance restent fragmentaires du fait des conditions difficiles de l'Occupation, ce manuscrit du poème «On dormait encore en ce temps-là » sur un feuillet plié en quatre qu'on peut cacher dans une poche est doublement émouvant : parce qu'il laisse imaginer l'arrière-texte d'un couple d'écrivains dans la clandestinité et parce que de l'inabouti d'une métaphore raturée ( «murmure, murmure ») naîtra bien plus tard, vingt ans après, une partie de la construction fictionnelle de La Mise à mort et de Blanche ou l'oubli comme romans de la mémoire du couple 9 . En offrant des graines pour les romans futurs - Aragon dans son discours du legs au CNRS a préféré la métaphore des pistes ( «J'imagine que si on pouvait examiner tout ce que j'ai écrit depuis l'enfance, on y trouverait bien des pistes pour ce que j'ai écrit plus tard») -, la poésie révèle ainsi sa fonction matricielle pour l'écriture d'Aragon dont l'avancée semble confirmer la thèse développée par Milan Kundera dans Le Rideau selon laquelle la genèse du romancier est toujours «l'histoire d'une conversion» car elle s'accomplit par le passage de la poésie (lyrique) au roman.

La démarche des chercheurs face à ce grand chantier de l'écriture que sont les manuscrits est à la fois quête possible de sens et conscience d'une irrépressible opacité, dualité paradoxale que le chercheur éprouve aussi face aux murs de la rue de Varenne ${ }^{10}$. Vitez disait dans le film de Reichenbach 11 : «Il y a des choses dans ces murs qui nous resteront à jamais incompréhensibles. » Murs et manuscrits, même constat... Celui-là même que fait Aragon dans l'épisode du film Dits et non-dits (1978) de Raoul Sangla consacré aux manuscrits : après avoir manifesté le souci de rendre «la rature lisible» pour les chercheurs, il exprime en même temps un certain scepticisme face à ces folios manuscrits du Fou d'Elsa surchargés de collages avec du scotch («ça se détache... c'est comme un puzzle, il faut recommencer à tout remettre en place, c'est ennuyeux...») et Jean Ristat n'est pas loin de lui faire avouer que ces manuscrits ne livrent pas le secret de la création, qui toujours excédera le travail des chercheurs.

9. Ces «splendides livres de vieillards » qu'Aragon en 1940 imagine écrire plus tard, comme il l'écrit à Mattew Josephson.

10. Voir notre article, p. 111.

11. L'appartement d'Aragon / Errer dans ces chambres, film de François Reichenbach et Monique Lepeuve, 1984.

MARYSE VASSEVIÈre a été maître de conférences à Paris 3 et chercheur au CNRS au sein de l'UMR «Écritures de la modernité». Elle travaille aujourd'hui, au sein de l'ERITA (Équipe de recherche interdisciplinaire sur Elsa Triolet et Aragon) et de l'équipe Aragon de l'ITEM, sur les questions de l'intertextualité (Aragon romancier intertextuel ou Les pas de l'étranger, L'Harmattan, 1998) et de la génétique, et sur la production journalistique d'Aragon considérée comme autant d'avant-texte de ses romans. Ses articles sont nombreux dans Recherches croisées Aragon Elsa Triolet, Mélusine, Revue des Sciences Humaines et Cahiers d'Aragon. 


\section{Résumés \\ Du manuscrit de poème aux germes du roman : graines, pistes et restes}

Après avoir étudié par le passé plusieurs des dossiers génétiques des romans d'Aragon, je tenterai encore une fois la démonstration de l'utilité d'étudier ses manuscrits légués au CNRS en 1977 et maintenant conservés à la $\mathrm{BnF}$ par une nouvelle étude de cas : le poème «On dormait encore en ce temps-là» du recueil de la poésie de la Résistance La Diane française (1944) dont on décrira le matériau génétique avant d'en donner une transcription diplomatique. Le deuxième temps de l'étude consistera à analyser le mouvement perpétuel de la genèse en dégageant quelques règles de l'avancée de l'écriture. Pour finir par mettre en évidence dans les graines de cet avant-texte poétique quelques restes, rejetés, inaboutis, inexplorés, et qui vingt ans plus tard vont permettre le passage de la poésie au roman et alimenter l'écriture romanesque ultime du dernier Aragon. Centré sur un des rares manuscrits de travail de la poésie de la Résistance, cet article pourrait être une nuance apportée à l'image d'un Aragon Jupiter de sa propre genèse.

After having already studied several genetic dossiers of Aragon's novels, I will attempt, once again, to demonstrate the usefulness of studying the manuscripts he bequeathed to the CNRS in 1977, which are now held at the BnF. I will present a new caste study: the poem "On dormait encore en ce temps là", from the book of poems, from the Resistance period, La Diane française (1944). We will describe the genetic material before presenting its diplomatic transcription. The second phase of our study will consist in the analysis of the genesis' perpetual movement, by determining some rules of the evolving writing. Finally we will show in these seeds of this poetic avant-texte a few left-overs, discarded, unfinished, unexplored, which, twenty years later, will allow the passage from the poem to the novel, and nourish the final fictional writings of the last Aragon. Centered on rare working manuscripts of the Resistance poetry, this article could bring a nuance to the image of a Aragon as Jupiter of his own genesis.

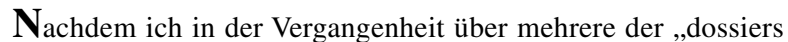
génétiques“ von Aragons Romanen gearbeitet habe, werde ich erneut versuchen, die Nützlichkeit der wissenschaftlichen Auswertung seiner 1977 dem CNRS vermachten und jetzt bei der Bibliothèque nationale de France aufbewahrten Manuskripte anhand einer neuen Fallstudie zu demonstrieren, und zwar geht es mir um das Gedicht „On dormait encore en ce temps-là“ aus der Sammlung der Widerstandsdichtung La Diane française (1944), dessen Textgenese beschrieben werden soll, bevor eine diplomatische Transkription davon gegeben wird. Der zweite Teil der Studie wird darin bestehen, die fortwährende Bewegung der Entstehung des Gedichts zu analysieren, indem einige Regeln für den Fortschritt des Schreibens identifiziert werden. Schließlich werden die Keime dieses poetischen avant-texte einige verworfene, unfertige, unerforschte Überreste enthüllen, die zwanzig Jahre später den Übergang von der Dichtung zum Roman ermöglichen und das letzte Romanschreiben des späten Aragons nähren werden. Dieser Artikel, der sich auf eines der seltenen Arbeitshandschriften der Dichtung des Widerstandes von Aragon konzentriert, könnte das Bild eines aragonischen Jupiters nuancieren, der über seine eigene Genese herrscht.

Después de haber estudiado varios dosieres genéticos de novelas de Aragon, intentaré una vez más demostrar la utilidad de estudiar sus manuscritos - donados al CNRS en 1977 y conservados actualmente en la $\mathrm{BnF}-$, analizando el poema «On dormait encore en ce temps-là» del libro de poemas de la Resistencia La Diane française (1944). En primer lugar, describiré el material genético y brindaré una transcripción diplomática del mismo; luego, analizaré el movimiento perpetuo de la génesis, deduciendo algunas reglas de la progresión de la escritura, y finalmente pondré de manifiesto en los granos de este pre-texto poético algunos restos, desechados, inacabados, inexplorados, que veinte años más tarde permitirán la transición de la poesía a la novela, alimentando la escritura novelesca postrera del último Aragon. Centrado en uno de los pocos manuscritos de trabajo de la poesía de la Resistencia, este artículo podría matizar la imagen de Aragon como demiurgo de la génesis propia.

Tendo anteriormente estudado vários dossiers genéticos dos romances de Aragon, tentarei mais uma vez, através de um novo estudo de caso, demonstrar a utilidade de estudar seus manuscritos legados ao CNRS, em 1977, e agora conservados na BnF. Trata-se do poema « On dormait encore en ce temps-là » da coletânea de poesia da Resistência, La Diane française (1944), do qual descreveremos o material genético antes da sua transcrição diplomática. Em seguida, este estudo consistirá em analisar o movimento perpétuo da gênese para extrair algumas regras da progressão da escrita. Por fim, evidenciará, nas sementes deste ante-texto poético, alguns restos, rejeitados, inacabados, inexplorados, que vinte anos mais tarde permitirão a passagem da poesia ao romance e alimentarão a derradeira escrita romanesca do último Aragon. Centrado num dos raros manuscritos de trabalho da poesia da Resistência, este artigo poderia introduzir um certo matiz na imagem de um Aragon Júpiter de sua própria gênese.

Dopo aver studiato in passato molti dossier genetici dei romanzi di Aragon, cercherò ancora una volta di dimostrare l'utilità di studiare i suoi manoscritti - donati al CNRS nel 1977 e oggi conservati alla $\mathrm{BnF}$ - analizzando il poema «On dormait encore en ce temps-là», tratto dalla raccolta di poesie della Resistenza La Diane française (1944). L'articolo descriverà in primo luogo il materiale genetico, presentando una trascrizione diplomatica. Successivamente, analizzerà il movimento perpetuo della genesi deducendo alcune regole di progressione della scrittura. Infine, metterà in evidenza, tra i semi di questo avantesto poetico, alcuni resti, rifiutati, incompleti, inesplorati, che permetteranno, venti anni più tardi, il passaggio dalla poesia al romanzo e nutriranno la scrittura romanzesca dell'ultimo Aragon. Centrato su uno dei rari manoscritti delle poesie della Resistenza, l'articolo potrebbe essere una sfumatura proposta all'immagine di un Aragon demiurgo della propria genesi. 